\title{
Improving continuous positive airway pressure adherence among adults
}

\author{
Christina Michele DiNapoli * \\ Weill Cornell Medical College Center for Sleep Medicine, New York, United States
}

Received: September 24, 2014

Accepted: November 21, 2014 Online Published: December 7, 2014

DOI: $10.5430 /$ jnep.v5n2p110

URL: http://dx.doi.org/10.5430/jnep.v5n2p110

\begin{abstract}
Study objectives: Continuous positive airway pressure (CPAP) is the gold standard of treatment for patients diagnosed with significant obstructive sleep apnea (OSA). It has been estimated that approximately $30 \%-50 \%$ of patients diagnosed with OSA reject CPAP immediately and $80 \%$ of patients prescribed CPAP are noncompliant within a year after starting CPAP therapy. Nonadherence with CPAP greatly reduces the overall effectiveness of treatment of OSA, leaving these patients at an increased risk for comorbid conditions, impaired daily functioning and decreased quality of life. The purpose of this study was to improve CPAP adherence by implementing an intervention that consisted of an education program and increased patient support options as well as increased provider education.

Methods: This study was a quasi-experimental study design. CPAP adherence rates on 660 patients at an academic sleep center in New York City were collected, 326 patients at baseline and 334 post-intervention. Interventions, included provider education on OSA and CPAP adherence; an improved and actively supported, individualized, patient education program; and additional individual and group support options were available for patients.

Results: Increased provider education, individualized intense patient education and patient support options increased CPAP adherence by $21 \%$. Increase in adherence between baseline and post-intervention group was statistically significant $(p=.005)$.

Conclusions: A targeted program of increased patient education and intensive patient support was effective at increasing CPAP adherence. The use of similar approaches may improve CPAP adherence in other centers. It is crucial to improve CPAP adherence among patients diagnosed with significant OSA in order to improve outcomes.
\end{abstract}

Key Words: Obstructive sleep apnea, Continuous positive airway pressure, Adherence, Compliance, Adult, Education, Sleep apnea, Positive airway pressure

\section{Introduction}

Obstructive sleep apnea (OSA) is a common sleep disorder that requires chronic care. Continuous Positive Airway Pressure (CPAP) is a highly effective treatment for OSA. CPAP delivers constant positive pressure to the airway through a mask, splinting the airway open throughout the night, eliminating breathing disruptions. ${ }^{[1,2]}$ CPAP is currently the first line treatment for patients with moderate to severe sleep apnea; yet despite its effectiveness, adherence with the treatment plan is poor. ${ }^{[1,2]}$ Proper education and follow up is extremely important for all patients; however, it is paramount for patients with OSA, as CPAP can be challenging to adapt to. There are various obstacles that CPAP patient's face that may lead to nonadherence, ranging

${ }^{*}$ Correspondence: Christina Michele DiNapoli; Email: chd9077@med.cornell.edu; Address: Weill Cornell Medical College Center for Sleep Medicine, New York, United States. 
from lack of proper education of diagnosis to physical discomfort from CPAP. It is estimated that $24 \%$ of the United States population suffers from OSA, however only about $4 \%$ have been diagnosed. ${ }^{[3,4]}$ The population of patients diagnosed with OSA is growing exponentially each year, a consequence of increased public awareness and provider education about OSA. ${ }^{[3,5,6]}$ Although CPAP is an extremely effective treatment for OSA, adherence is a critical problem and is widely recognized as a significant limiting factor in successful treatment. ${ }^{[4]}$ Research shows that $30 \%-50 \%$ of patients diagnosed with OSA, where CPAP is recommended, reject CPAP immediately and approximately $80 \%$ of CPAP patients are noncompliant within a year after starting CPAP therapy. ${ }^{[7]}$ Nonadherence with CPAP significantly reduces the overall effectiveness of treatment of OSA, leaving these patients at an increased risk for comorbid conditions, impaired daily functioning, and decreased quality of life. ${ }^{[2]}$

OSA has a significant impact on patients if not treated properly. Untreated significant OSA is associated with an increased risk of cardiovascular and cerebrovascular diseases. ${ }^{[3]}$ Untreated, it leaves patients with a three-fold increase in risk for hypertension, diabetes mellitus and stroke. ${ }^{6]}$ Patients are also at an increased risk for ischemic heart disease, cardiac arrhythmia, congestive heart failure, peripheral vascular disease and valvular cardiac disease. ${ }^{[8]}$ Excessive daytime sleepiness, a consequence of the sleep fragmentation due to recurrent airway closures, has been shown to adversely impact quality of life. ${ }^{[3,6]} \mathrm{Pa}-$ tients with untreated OSA often experience excessive daytime sleepiness, impaired cognition and memory, decreased functional and occupational capacity as well as mood alterations, which significantly decrease quality of life. ${ }^{[3,6]} \mathrm{Be}-$ sides affecting the patients quality of life and health outcomes, it has been shown that untreated OSA patients have up to two times greater health care costs than similar individuals without OSA. ${ }^{[9]}$

Improvements in CPAP adherence would positively impact patients physiologically, psychologically and socially. ${ }^{[3,6]}$ By improving CPAP adherence, patients may decrease their risk of comorbid conditions associated with untreated OSA. ${ }^{[6]}$ The patient's quality of life, cognitive function and occupational function would also improve, as daytime symptoms decrease when effectively treated by CPAP. ${ }^{[3,6]}$ With increased CPAP adherence, patients may experience less hypersomnia, consequently reducing their increased risk of accidents and poor job performance. It has been estimated that 567,000 collisions and 980 fatalities annually could be avoided by effective CPAP treatment. ${ }^{[10]}$ Additionally, effective treatment of OSA will decrease health care system costs as it decreases the care required for associated aforementioned comorbitities. ${ }^{[9]}$ It is extremely important to implement the evidence-based practice changes that are most effective in increasing CPAP adherence among adult patients diagnosed with OSA.

Published by Sciedu Press

\subsection{Literature review}

A literature review was undertaken to better understand the most effective published method of increasing CPAP adherence. Many mechanical, psychological and educational interventions have been proposed to increase CPAP adherence in patients diagnosed with OSA. The search terms that were used were "Obstructive Sleep Apnea", "Continuous Positive Airway Pressure", "Nonadherence" and "Noncompliance".

After the search was completed a total of 8,618 articles were produced. The articles were then evaluated for duplicates, English language and inclusion/exclusion criteria. After the review, 28 articles were left for evaluation.

\subsection{Synthesis of evidence}

After the evaluation of each article two overall themes were identified in improving CPAP adherence in adults diagnosed with OSA: increased patient education and intensive patient support. ${ }^{[11]}$ The delivery of intensive patient education was looked at in many different ways. ${ }^{[13-17]}$ Within each method of communicating patient education information there were three parts: the diagnosis of OSA, the treatment of OSA and remedies to CPAP problems that patients may experience. Education was given on the definition and pathophysiology of OSA. The procedure to diagnose OSA was also highlighted. Side effects and consequences of untreated OSA were communicated to patients at length. The consequences of untreated OSA that were targeted included health risks as well as personal costs. The purpose and definition of CPAP was then highlighted. Finally, remedies to common CPAP problems were discussed at length. Overall, the evidence showed a strong link to intensive patient education and increased CPAP adherence. ${ }^{[11,13-17]}$

The next theme of improving CPAP adherence was intensive patient support. Each type of intensive support had similar activities that were performed during these sessions. ${ }^{[18-22]}$ The activities consisted of CPAP adherence, assessing for the presence of any side effects with CPAP, equipment review and finally, a mask fitting was performed. Overall patient education and intensive support were the two most effective interventions shown to increase CPAP adherence. ${ }^{[11,18-22]}$

\section{Methods}

\subsection{Study design}

This was a quasi-experimental study design and there were two research questions: 1) Will the interventions of increased patient education and increased patient support increase CPAP adherence? 2) Will an educational program geared towards the providers increase provider's knowledge of CPAP treatment? The study was conducted at an academic sleep center in New York City. The purpose of this study was to improve CPAP adherence among adult patients 
at the sleep center. The intervention consisted of an individualized patient education program, increased patient support options including a CPAP Clinic and CPAP group support session as well as a provider education session. ${ }^{[1]}$ Prior to the intervention, 326 patients seen between October 2012 and December 2012, who were diagnosed with OSA and prescribed CPAP served as the baseline group. After the intervention was implemented CPAP adherence was evaluated in a group of 334 patients between November 2013 and January 2014. For the purpose of this study CPAP adherence will be defined as $70 \%$ usage for over four hours per night in any given thirty day period which is tracked internally in CPAP devices. This definition was chosen, as it is the definition that Medicare uses to define CPAP adherence. ${ }^{[12]}$

\subsection{Participants and procedure}

There were 326 patients prescribed CPAP, between October 2012 and December 2012, serving as comparison participants, and 334 patients prescribed CPAP, between November 2013 and January 2014, serving as intervention participants. Potential participants had a history and physical, as well as objective testing which was either an inlaboratory polysomnography (PSG) or home testing with portable monitors (PM). The diagnosis of sleep apnea was confirmed if the number of obstructive respiratory events on the PSG or PM was greater than 15 events per hour or greater than five events per hour with reports of hypersomnia, unrefreshing sleep, fatigue, gasping or choking for air at night. ${ }^{[1]}$ All patients who met the inclusion criteria of a diagnosis of OSA, had been advised to use CPAP as their treatment for OSA during these time periods, and were over the age of 18 were included as participants.

The medical center's Institutional Review Board deemed the study as "exempt" as it was a quality improvement study. There were 650 charts which were reviewed for the study. The patients' charts were reviewed to obtain the following variables: CPAP adherence, age, sex, type of CPAP mask worn, provider, and date of CPAP titration. All variables were measured in a three-month time frame from before the intervention as well as three months post intervention. CPAP adherence was the outcome measure, which was collected for all CPAP patients who were seen at the sleep center between October 2012 and December 2012. The participant's demographics for pre and post-intervention are provided in Table 1.

Table 1: Patient population characteristics at baseline and post intervention-Mean (SD) or Percent

\begin{tabular}{|c|c|c|c|}
\hline & $\begin{array}{l}\text { Baseline Group } \\
(\mathrm{n}=326)\end{array}$ & $\begin{array}{l}\text { Intervention Group } \\
(\mathrm{n}=334)\end{array}$ & $p$ \\
\hline \multicolumn{4}{|l|}{ Demographics } \\
\hline Age & $60.1(14.70)$ & $60.7(14.10)$ & .633 \\
\hline Female & $32.2 \%$ & $28.4 \%$ & .293 \\
\hline Male & $67.8 \%$ & $71.6 \%$ & .293 \\
\hline Length of time on CPAP (yr) * & $2.8(2.34)$ & $1.7(1.74)$ & .000 \\
\hline \multicolumn{4}{|l|}{ Documentation of Mask Type } \\
\hline Nasal Pillows & $29.8 \%$ & $30.8 \%$ & .762 \\
\hline Nasal Mask* & $15.6 \%$ & $28.4 \%$ & .000 \\
\hline Full Face Mask* & $8.3 \%$ & $15.9 \%$ & .003 \\
\hline Not Documented* & $46.3 \%$ & $24.9 \%$ & .000 \\
\hline
\end{tabular}

* Statistically significant $p<.05$ (continuous variables tested with independent $t$-test and proportions tested with chi square)

Table 1 looks at the distribution of the demographics in the pre and post-intervention participants. The distribution of patient age, sex, length of time on CPAP (in years), and documentation of mask type.

Table 1 compares the intervention and comparison patient group characteristics. There was no significant difference between patient ages or patient gender between the intervention and comparison group. The length of time on CPAP in the comparison and intervention group was compared using an independent $t$ test. The comparison group had a mean of 2.83 years on CPAP, and the intervention group's mean of 1.67 years on CPAP $(p<.001)$. There was also a significant difference between groups on patient mask type (compared with a chi square), likely due to the increase in documentation in the post-intervention group. In the comparison group $43.7 \%$ of charts had documentation of type of mask used as compared to $75.1 \%$ in the intervention group $(p<.000)$.

The providers at the sleep center were also targeted, which consisted of two full time and two part time medical doctors, two full time doctors of psychology, and two full time nurse practitioners were given and educational session to improve knowledge of OSA and CPAP. The practitioners' participation was voluntary and no identifying or demographic information was collected. 


\subsection{Intervention protocol}

All patients who were using CPAP during the intervention period of October 2013 through January 2014 were given an individualized patient education program. This program included education given by the provider as well as an overview of OSA and CPAP in a brochure specifically created for patients with OSA and using CPAP. Detailed education was provided on the definition, pathophysiology and diagnosis of OSA, side effects and consequences of untreated OSA, the purpose and definition of CPAP, and remedies to common CPAP problems were all discussed.

All patients during the intervention time period were also offered individualized CPAP support, which was termed a "CPAP clinic appointment". The patient could request a CPAP clinic appointment or a provider could request that the patient have a CPAP clinic appointment. The CPAP clinic appointments were focused only on OSA and CPAP, and served as an "urgent care" for CPAP users. During the CPAP clinic visit the provider discussed all of the following that pertained to the patient: OSA, CPAP, CPAP adherence, remedies to CPAP issues, ways to improve CPAP adherence, mask fitting, review of machine and equipment and answered all patient questions. The CPAP clinic sessions were held twice a week, during designated times, if additional time was needed it was scheduled at the patients convenience.

A CPAP group support session was also offered to all patients using CPAP, free of charge. The support session was held after work hours at the sleep center and was moderated by the two nurse practitioners. Patients were able to have open conversations about OSA and CPAP with providers as well as other patients who are using CPAP. If a patient had an individual issue that came up, it was addressed with the group if possible, and if not a CPAP clinic appointment was made.

The second intervention consisted of a provider educational session. Each provider was asked to fill out an anonymous assessment of knowledge of OSA and CPAP before the intervention. The assessment was emailed to all providers, and the provider was asked to fill out the assessment electronically and anonymously. The provider was asked to print the assessment and put it in a locked box. The assessments were scored by percent of "always" answers chosen. A provider educational session was then given at a mandatory provider meeting. After the meeting the same assessment was redistributed by email. Each provider completed the assessment anonymously and electronically and placed it in a locked box inside a locked office. The assessments were again scored using the same criterion.

\subsection{Statistical analysis}

Descriptive statistics were used to analyze and compare CPAP adherence between the comparison and intervention groups. The dependent variable was the percentage of patients who were effectively managing their OSA, and the independent variable was the total number of patients. A chi square test was used to evaluate the difference between the intervention and comparison group. In order to evaluate the provider's assessment scores pre and post-intervention each assessment was analyzed individually and then combined. The dependent variable was the providers' mean assessment score and the independent variable was the number of providers who participated. Statistical testing could not be completed as the provider assessments were not paired.

\section{Results}

There were a total of 660 patients included in the study, 326 patients in the comparison group and 334 patients in the intervention group. To evaluate adherence, each of the 660 patient's charts was evaluated. Patient's age, gender, CPAP adherence status, type of CPAP mask, length of time on CPAP as well as patient provider was evaluated (see Table 1). There were no significant differences between the comparison and intervention patient groups in the categories of age of patient $(p=.633)$ or gender of patient $(p=.293)$. There was a significant difference in length of time on CPAP, the distribution of patient providers between the comparison and intervention groups as well as the documentation of the type of mask worn by patients between the comparison and intervention group. Patient adherence status was evaluated in both the comparison and intervention groups; $54.6 \%$ of patients were compliant with CPAP in the comparison group and $65.9 \%$ of the intervention patients were compliant with CPAP (see Figure 1). A chi square test of proportions was used to analyze the difference in CPAP adherence between the comparison and intervention groups, which was significant $(p=.005)$. An improvement in CPAP adherence in the intervention group demonstrates the intervention improved CPAP adherence.

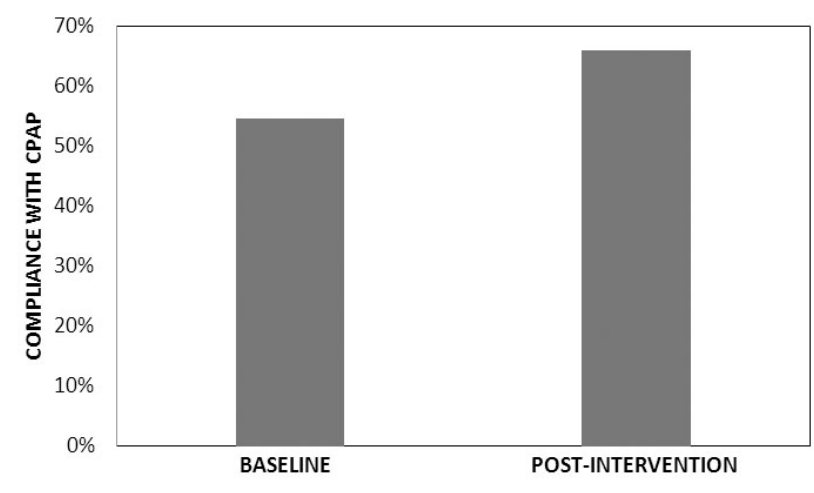

Figure 1: CPAP at baseline and post-intervention

Figure 1 shows the CPAP adherence in both the comparison and intervention groups. In the comparison group 54.6\% of patients were compliant with CPAP. In the intervention 
group $65.9 \%$ of patients were compliant with CPAP. After the intervention there was a $21 \%$ increase in CPAP adherence $(p=.005)$.

Direct logistic regression was performed to assess the impact of patient length of time on CPAP, patient mask type as well as patient provider on the likelihood that participants would be compliant with CPAP (see Table 2). The full model containing all the predictors was statistically significant, $\chi^{2}(8, \mathrm{~N}=660)=55.20, p \leq .001$, indicating that the model was able to distinguish between participants who were compliant and noncompliant with CPAP. The model as a whole explained between $6.7 \%$ (Cox \& Snell R Square) and 9\% (Nagelkerke R Square) of the variance in compliance, and correctly identified $65 \%$ of the cases. As shown in Table 2 only two variables made a uniquely statistically significant contribution to the model (length of time on CPAP and patient provider). The strongest predictor of compliance, recording an odds ratio of 3.7 was provider 1, indicating that the patients whom saw provider 1 were over 3 times more likely to be compliant with CPAP controlling for other factors in the model. The odds ratio for length of time on CPAP was 1.1 indicating that patients who were on CPAP longer were 1.1 times more likely to be compliant with CPAP, controlling for other factors in the model. After adjusting for confounders the intervention remained effective in significantly improving CPAP compliance post intervention.

Table 2: Logistic regression predicting likelihood of patient being compliant with CPAP

\begin{tabular}{lllllll}
\hline & B & S.E. & Wald & Df & $P$ & $\begin{array}{l}\text { Odds } \\
\text { Ratio }\end{array}$ \\
\hline $\begin{array}{l}\text { Length of time } \\
\text { On CPAP (yrs) }\end{array}$ & .124 & .046 & 7.172 & 1 & .007 & 1.132 \\
$\begin{array}{l}\text { Type of CPAP } \\
\text { Mask }\end{array}$ & & & 2.232 & 3 & .526 & \\
Patient Provider & & & 28.998 & 6 & .000 & \\
Intervention & -.640 & .188 & 11.643 & 1 & .001 & .527 \\
Constant & -.020 & .488 & .002 & 1 & .968 & .980 \\
\hline
\end{tabular}

Table 2 shows the results of the logistic regression that was performed to assess the impact of variables (length of time on CPAP (in years), type of patient mask as well as patient provider) on compliance.

There were six providers included in the study; descriptive data was not obtained for the provider group. To evaluate the knowledge improvements in providers an assessment was completed both pre and post-intervention. As the assessments were not paired statistical analysis could not be completed. The provider's average assessment score pre-intervention was $70 \%$ and the post-intervention average score was $86.67 \%$; which was a $23.8 \%$ increase in the mean of the providers score. In evaluating the type of masks between the pre and post-intervention group a significant difference was seen in the documentation of masks worn by patient. In the pre-intervention group $53.7 \%$ of patients included did have type of CPAP mask used documented in their chart as compared to $75.1 \%$ in the post-intervention group, which showed a significant difference $(p<.000)$ (see Figure 2).

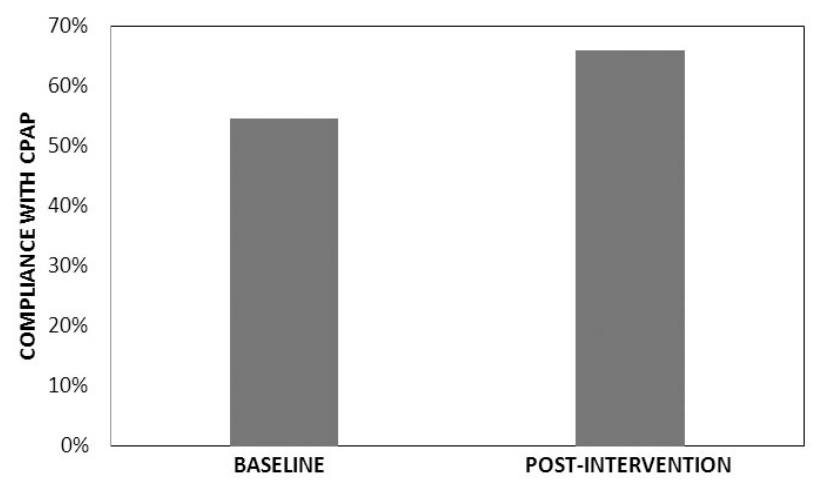

Figure 2: Mean assessment score on the assessment

Graph 2 shows the provider mean assessment score pre and post-intervention. The mean assessment score preintervention was $70 \%$ and $86.7 \%$ in the post-intervention group.

\section{Discussion}

This study found significant improvement in CPAP adherence in patients who received the intervention of intensive patient education, increased patient support options as well as a provider education session, as compared to the comparison group $(p=.005)$. This finding is consistent with what is currently in the literature. Many studies have shown significant increases in CPAP adherence after an increased education program was given to CPAP patients. ${ }^{[13-17]}$ Multiple studies have also shown that increased patient support significantly increases CPAP adherence. ${ }^{[11,18-22]}$ Currently, there are not any guidelines or policies in place for sleep centers to implement in order to improve patient adherence with CPAP. After searching the literature there were various different articles on interventions which have been shown to improve CPAP adherence, however very few have been replicated and even fewer were generalizable. As untreated sleep apnea is not only detrimental to a patient's health but it also can affect the health care system as a whole as well, it is imperative that research on interventions to improve CPAP adherence be conducted, replicated and disseminated.

This study also found improvement in provider knowledge of OSA and CPAP after a focused educational session. This demonstrated the necessity of continued provider education. Currently, there is not a policy stating the frequency that the provider must attend continued education in their specialty, however this is something that should be further evaluated as it may improve both provider knowledge as well as patient outcomes. 
A limitation of this study was that a provider was on leave during the post-intervention time period and therefore only six out of the seven providers $(85.7 \%)$ were able to participate in the intervention. The assessments given to providers were not paired therefore in did not allow for statistical testing which was another limitation. As a provider was out of the office each of the other providers had some increased responsibility, which may have underestimated the effect of the study. Another potential limitation of the study was that, originally CPAP group support sessions were to be held monthly, however due to time constraints, only one was held during the intervention time period. Although one session was held it was very well received and well attended by patients. The time intervals to measure CPAP adherence may not have been sufficient to see the full benefit of the intervention, and it is also plausible that CPAP adherence may have increased at a larger percent if the subjects in comparison and intervention group had been the same. Lastly, as this study was a held in a single setting it is not generalizable to all sleep centers.

\section{Conclusion}

CPAP is an effective treatment for OSA, however adherence to CPAP is often low which puts patients at an increased risk for comorbid conditions, increased health care costs and decreased quality of life. An intervention aimed at patients and providers, focusing on increased patient education and intensive patient support, was shown to significantly increase CPAP adherence. Nurses can have a large role in providing provider education, patient education as well as patient support. It is essential to empower nurses to help implement these programs in order to effectively treat patients whom are diagnosed with OSA. Further research should aim to create programs which other sleep centers could implement to increase CPAP adherence. In addition, it is also necessary to carry out longitudinal studies to look at the long-term effects of these interventions.

\section{Acknowledgements}

The author would like to thank Dr. Anne Belcher, her academic mentor at Johns Hopkins University, Dr. Kathleen Becker, her course coordinator as well as her organizational mentor Dr. Ana Krieger for their help and guidance during this process.

\section{Conflicts of Interest Disclosure}

The author declares that there is no conflict of interest statement.

\section{References}

[1] Epstein, L., Kristo, D., Strollo, P., et al. Clinical Guideline for evaluation, management and long-term care of obstructive sleep apnea in adults. J Clin Sleep Med. 2009; 5(3): 263-276. PMID:19960649

[2] Weaver, T., Sawyer, A. Adherence to continuous positive airway pressure treatment for obstructive sleep apnea: Implications for future interventions; Indian J Med Res. 2010; 131: 248-258.

[3] Siccoli, M., Pepperell, J. Kohler, M., Craig, S., Davies, R., Stradling, J. Effects of continuous positive airway pressure on quality of life in patients with moderate to severe obstructive sleep apnea: Data from a randomized control trial. Sleep. 2008; 31(11): 1551-1558. PMID: 19014075

[4] Weaver, T., Grunstein, R. Adherence to continuous positive airway pressure therapy. Proc Am Thorac Soc. 2008; 5: 173178. PMID:18250209 http://dx.doi.org/10.1513/pats.20 0708-119MG

[5] American Academy of Sleep Medicine; Positive airway pressure (PAP) devices-Physician frequently asked questions. 2008.

[6] Young, T., Peppard, P., Gottlieb, D. Epidemiology of obstructive sleep apnea. Am J Respir Crit Care Med. 2002; 165: 12171239. PMID:11991871 http://dx.doi.org/10.1164/rccm.21 09080

[7] Weaver, T., et al. Relationship between hours of CPAP use and achieving normal levels of sleepiness and daily functioning. Sleep. 2007; 30(6): 711-719. PMID:17580592

[8] Simon-Tuval, T., Reuveni, H., Greenberg-Dotan, S., Oksenberg, A., Tal, A., Tarasiuk, A. Low socio economic status is a risk factor for CPAP acceptance among adult OSAS patients requiring treatment. Sleep. 2009; 32(4): 545-552. PMID:19413149
[9] Wittmann, V., Rodenstein, D. Health care costs and sleep apnea syndrome. Sleep Med Rev. 2004; 4: 269-279. PMID:15233955 http://dx.doi.org/10.1016/j.smrv.2004.01.002

[10] Rodenstein, D. Sleep apnea: Traffic and occupational accidentsindividual risks, socioeconomic and legal implications. Respiration 2009; 78: 241-248. PMID:19494476 http://dx.doi.org/10.11 59/000222811

[11] DiNapoli, C: Strategies to improve continuous positive airway pressure adherence: A review. Journal of Nursing Education and Practice. 2014; 4(7): 62-72. http://dx.doi.org/10.5430/jnep.v4 n7p62

[12] American Academy of Sleep Medicine. Positive airway pressure (PAP) devices- Physician frequently asked questions. 2008.

[13] Ballard, R. D., Gay, P. C., Strollo, P. J. Interventions to improve compliance in sleep apnea patients previously non-compliant with continuous positive airway pressure. Journal of Clinical Sleep Medicine: JCSM : Official Publication of the American Academy of Sleep Medicine. 2007; 3(7): 706-712. PMID: 18198804

[14] Smith, I., Lasserson, T., Haniffa, M. Interventions to improve use of continuous positive airway pressure for obstructive sleep apnoea (review). The Cochrane Collaboration. 2009; (3).

[15] Chervin, R. D., Theut, S., Bassetti, C., Aldrich, M. S. Compliance with nasal CPAP can be improved by simple interventions. Sleep. 1997; 20(4): 284-289. PMID:9231954

[16] Fuchs, F. S., Pittarelli, A., Hahn, E. G., Ficker, J. H. Adherence to continuous positive airway pressure therapy for obstructive sleep apnea: Impact of patient education after a longer treatment period. Respiration; International Review of Thoracic Diseases. 2010; 80(1): 32-37. PMID:19786730 http://dx.doi .org/10.1159/0 00243161

[17] Golay, A., Girard, A., Grandin, S., et al. A new educational program for patients suffering from sleep apnea syndrome. Patient Ed- 
ucation and Counseling. 2006; 60(2): 220-227. PMID:16253467 http://dx.doi.org/10.1016/j.pec. 2005.01.007

[18] Hoy, C. J., Vennelle, M., Kingshott, R. N., Engleman, H. M., Douglas, N. J. Can intensive support improve continuous positive airway pressure use in patients with the sleep apnea/hypopnea syndrome? American Journal of Respiratory and Critical Care Medicine. 1999; 159(4 Pt 1): 1096-1100. PMID:10194151 http://dx.doi.org/1 0.1164 /ajrccm.159.4.9808008

[19] Damjanovic, D., Fluck, A., Bremer, H., Muller-Quernheim, J., Idzko, M., Sorichter, S. Compliance in sleep apnoea therapy: Influence of home care support and pressure mode. The European Respiratory Journal: Official Journal of the European Society for Clinical Respiratory Physiology. 2009; 33(4): 804-811. PMID:19129293 http://dx.doi.org/10.1183/09031936.00023408

[20] DeMolles, D. A., Sparrow, D., Gottlieb, D. J., Friedman, R. A pi- lot trial of a telecommunications system in sleep apnea management. Medical Care. 2004; 42(8): 764-769. PMID: 15258478 http: //dx.doi.org/10.1097/01.mlr.0000132353.99209.fe

[21] Richards, D., Bartlett, D. J., Wong, K., Malouff, J., Grunstein, R. $\mathrm{R}$. Increased adherence to CPAP with a group cognitive behavioral treatment intervention: A randomized trial. Sleep. 2007; 30(5): 635640. PMID: 17552379

[22] Roecklein, K., Schumacher, J., Gabriele, J., Fagan, J., Baran, A., Richert, A. Personalized feedback to improve CPAP adherence in obstructive sleep apnea. Behavioral Sleep Medicine. 2010; 8: 105112. PMID:20352546 http://dx.doi.org/10.1080/1540200 1003622859

[23] Boss, C. Searching for evidence: mission-critical tips. Nursing Management. 2009; 40(9): 12-14. PMID:19734750 http://dx.doi.o rg/10.1097/01. NUMA.0000360766.29235.8e 